\title{
Communication organisationnelle
}

Il n'y a pas de projet sans connaissance de l'identité

\section{Béatrice Galinon-Mélénec}

\section{(2) OpenEdition}

\section{Journals}

Édition électronique

URL : http://journals.openedition.org/communicationorganisation/1542

DOI : 10.4000/communicationorganisation. 1542

ISSN : 1775-3546

Éditeur

Presses universitaires de Bordeaux

Édition imprimée

Date de publication : 1 mai 1992

ISSN : 1168-5549

\section{Référence électronique}

Béatrice Galinon-Mélénec, " Communication organisationnelle », Communication et organisation [En

ligne], 1 | 1992, mis en ligne le 26 mars 2012, consulté le 05 mai 2019. URL : http://

journals.openedition.org/communicationorganisation/1542 ; DOI : 10.4000/

communicationorganisation. 1542

Ce document a été généré automatiquement le 5 mai 2019.

(c) Presses universitaires de Bordeaux 


\title{
Communication organisationnelle
}

Il n'y a pas de projet sans connaissance de l'identité

\author{
Béatrice Galinon-Mélénec
}

1 Les expressions "se projeter ", «avoir un projet», renvoient immédiatement à la question de savoir qui se projette, qui a un projet.

2 Et la réponse suppose d'avoir résolu en préalable le problème de l'identité.

\section{La personne:}

3 En ce qui concerne les individus, Paul Ricoeur (1990) ${ }^{1}$ rappelle qu'il faut distinguer deux significations majeures de l'identité.

4 En effet, le mot « identité » - dont le terme voisin « identique » renvoie au sens de même - peut désigner :

- tantôt la permanence dans le temps ${ }^{2}$

- tantôt l'expérience intime de soi ${ }^{3}$.

5 Pour cet auteur, « le concept de personne n'est pas moins une notion primitive que celui de corps ; il ne s'agira pas d'un second référent distinct du corps, telle l'âme cartésienne, mais, d'une manière qui restera à déterminer, d'un unique référent doté de deux séries de prédicats, des prédicats physiques et des prédicats psychiques $»^{4}$.

6 En fait ce sont les corps qui sont perceptibles au tiers et c'est leur émergence puis leur permanence qui est assimilé à l'existence ${ }^{5}$; c'est leur identification et leur ré identification qui assure que nous sommes bien en face d'une même personne (vivante).

7 Ainsi, le corps par l'objectivation de l'être qu'il représente apparaît comme la perception première d'une personne. Ses pensées, ses représentations par leur vécu profondément intime correspondent à "une boîte noire » dont la compréhension est difficilement accessible au tiers ${ }^{6}$.

8 En même temps, chacun est imprégné dans son expérience intérieure du " COGITO ERGO SUM $»^{78}$ et pense la conscience comme première ; le corps semble alors abriter la pensée, 
l'extérioriser (il manifeste les sentiments, les sens captant les données qui seront « incorporées)». Pour Descartes, le sujet existe comme conscience de soi, comme acte de pensée ; il se sait existant par le fait même qu'il se pense, qu'il se pense pensant.

9 Selon Sartre, "l'homme n'est rien d'autre que ce qu'il fait»; la chose existe en ellemême, elle n'est ni moi ni l'autre ; elle seule dépasse la subjectivité pour exister en dehors des regards humains. L'homme n'est rien d'autre que ce qu'il fait, à condition qu'il ait la conscience de l'unité des faits qu'il a produits. L'identité n'est pas dans l'extériorité des effets, elle est dans la mise en relation qui donne sens - aux yeux de leur auteur - à leur succession.

Cette conception a une influence sur la perception du projet : le plus souvent la question $\mathrm{du}$ « qui » pose moins question que le « quoi ».

11 Ce primat de la chose prend une ampleur encore plus grande, quand, passant de l'individuel au collectif, on s'occupe du projet des organisations.

\section{L'organisation $^{9}$}

12 Il s'agit pour nous de tenter un transfert des distinctions corps-pensée préalablement examinées à propos de l'identité d'une personne sur les organisations.

13 L'organisation peut être considérée comme une entité - économique, juridique, par exemple - indépendante des hommes qui la composent mais elle ne peut exister sans eux. Il est commun de lui prêter des caractéristiques semblables à celles des êtres humains ; ainsi on parlera de la « mort » d'une entreprise, de son « identité », de sa " personnalité », de sa « culture », de sa « responsabilité », de son « éthique », de son projet ${ }^{10}$.

Mais ce transfert s'effectue le plus souvent sans s'interroger sur les fondements des concepts. Ainsi, pour reprendre l'exemple que nous examinons ici, le concept d'identité peut se référer soit à l'extériorité des corps soit à la perception intérieure d'existence et de conscience.

En général, l'organisation favorise le tangible, le quantifiable, le dénombrable, l'évaluable, - et non l'immatériel - pour définir l'identité. Mais l'on pourrait imaginer de prendre en compte de façon aussi puissante, la " pensée collective ».

Dans ce contexte, le projet d'une organisation ne peut se réduire à la question du " quoi » (quel projet pour telle organisation? on parlera de projet-programme), mais doit d'abord passer par la question du « Qui » (quelle est l'identité de l'organisation qui se projette ; on parlera de projet organisationnelle).

17 Cette définition de l'identité est du plus grand intérêt car elle suppose que l'organisation valorise la " pensée $»^{11}$ (et pas seulement les actes, la production) des personnes humaines qui la composent.

Pour transposer l'idée de Descartes à l'organisation, nous pouvons tenter d'énoncer que l'organisation existe en tant que conscience d'elle-même à condition de concevoir cette conscience comme acte de pensée et non comme substance.

Dans ce contexte, la communication des organisations prend une place essentielle : elle devient un préalable indispensable. Si l'on admet que l'organisation puisse avoir une identité, on ne peut, sans risque de vider le mot de son sens, échapper à la nécessité de la cohérence de pensée de cette unité. 
20 Pour reporter ici ce que nous avons conclu à propos de la position de Sartre sur la relation faits-identité, nous dirons que l'identité de l'organisation apparaît moins dans l'énoncé de la juxtaposition de ses actes que dans la conscience de leur unité, et à travers elle, dans l'organisation se pensant en tant que entité-sujet consciente de la " grammaire génératrice $»^{12}$ de ses comportements ;

21 Celle-ci ne se décrète pas. Elle émerge lentement par une communication interactive multidirectionnelle (aussi bien ascendante que descendante ou latérale). Elle est le ciment de l'organisation, elle en assure l'harmonie.

22 P.Schwebig ${ }^{13}$, dès 1988, présentait l'importance de la relation identité-projetcommunication d'une organisation. Mais la force de ce rapprochement n'a peut-être pas été totalement perçue. Comme lui, nous pensons que le concept d'identité est fondamental. Et il ne devrait plus être possible pour une organisation de parler de sa "mémoire» de sa "culture ", de son "éthique», de ses "valeurs" sans avoir au préalable - pour des raisons logiques - précisé son identité. En somme, l'identité est à construire, elle n'est pas donnée d'emblée. Elle est première logiquement comme principe d'unité et d'horizon.

\section{Conclusion}

Il paraît difficile en matière organisationnelle d'éviter la question du « qui » agit. Si l'organisation est perçue comme une entité, celle-ci doit être définie non seulement à partir de critères quantifiables mais aussi en terme de "pensée collective". Il nous semble qu'une telle approche est porteuse de valeur ajoutée à la fois sur un plan conceptuel et pratique. Elle suppose un investissement préalable en terme de temps passé à la communication interne et à l'apprentissage de modes de relations nouveaux. Elle est susceptible, d'une part, de constituer un apport décisif dans la relation entre l'acteur et l'organisation qui l'emploie ${ }^{1415}$ et d'autre part de modifier profondément la conception que l'organisation a d'elle-même ${ }^{16}$.

\section{NOTES}

1. « soi-même comme un autre » 1990, Paris,Seuil, p. 12.

2. L'idem : le même

3. L'ipse : soi-même

4. P. Ricœur, ibid, p. 46

5. Notons néanmoins que les morts gardent leur Identité.

6. Sauf il en existe une objectivation par l'écriture.

7. Descartes Méditations métaphysiques, Paris, Garnier-Flammarion.

8. Discours de la méthode.

9. Tout regroupement organisé de personnes : exemples l'entreprise, l'université...

10. Pour ne citer que quelques aspects. 
11. Si nous sortions de la comparaison initiale (corps-pensée) on pourrait aussi étudier d'autres aspects immatériels dont les émotions, l'affectif.

12. "Grammaire génératrice des comportements", expression utilisée par le sociologue P. Bourdieu ; ici nous l'utilisons pour signifier que la relation entre identité et comportements peut être comparée à celle existant entre la grammaire -dont les règles sont relativement peu nombreuses et structurantes de l'expression - et la multitude de phrases -exprimant en toute liberté la pensée que l'on peut énoncer en s'y conformant.

13. Schwebig Les communications de l'entreprise ; au-delà de l'image, 1988, Paris, Mac CrawHill.

14. CF M. Crazier, E. Friedberg L'acteur et le système. Parts, Editions du Seuil, 1977, p. 440

15. Pour la gestion de la relation entre projet individuel et projet collectif, cf B. Galinon-Ménélec Projet et communication dans les universités, 1991, Paris, Editions d'organisation. Signalons simplement qu'il existe des différences culturelles dans cette gestion (à cet égard, la comparaison France-Japon est significative)

16. L'identité de l'organisation est un tout en soi d'un degré de complexité supérieur au degré de complexité de ses parties et de la somme des identités des personnes qui la composent. Elle a des propriétés propres. Mais elle est néanmoins dépendante des composantes.

\section{RÉSUMÉS}

Il est commun de prêter aux organisations des caractéristiques semblables à celles des êtres humains. Ainsi on parlera de la «mort» de l'entreprise, de son «identité », de sa "personnalité », de son « éthique », de son " projet ». Ce transfert s'effectue le plus souvent sans s'interroger sur les fondements des concepts. L'auteur ouvre ici des pistes en ce qui concerne le lien entre projet et identité.

\section{AUTEUR}

\section{BÉATRICE GALINON-MÉLÉNEC}

Maître de conférences à l'ISIC, Université Michel de Montaigne - Bordeaux 3 et chercheur au GREC/O, spécialiste des méthodes de conduite de projet, elle a publié plusieurs études sur cette question et notamment Projet et communication dans les universités. Éditions d'organisation, Paris, 1991. 